Radial and Nonradial Pulsations as Probes of Stellar Physics

ASP Conference Series, Vol. 259, 2002

C. Aerts, T.R. Bedding, \& J. Christensen-Dalsgaard, eds.

\title{
Velocity Observations of Multiple Mode AGB Variable Stars
}

\author{
T. Lebzelter \\ Institute for Astronomy, Türkenschanzstrasse 17, A1180 Vienna, \\ Austria
}

\author{
K.H. Hinkle, R.R. Joyce \\ National Optical Astronomy Observatory, P.O. Box 26732, Tucson, AZ \\ 85726-6732 \\ F.C. Fekel \\ Tennessee State University, Center of Excellence in Information \\ Systems, 330 10th Avenue North, Nashville, TN 37203
}

\begin{abstract}
Numerous infrared spectroscopic observations were obtained of eight AGB field M giants that have multiple periods of light variability. For six of the eight giants we found radial-velocity periods that confirm the long-period light variability. Although we consider the possibility that the velocity variations result from orbital motion, we conclude that the long-period velocity changes in most, if not all of our sample stars, likely result from pulsation rather than duplicity.
\end{abstract}

A number of late-type giant variables have been found to have at least two periods (e.g. Houk, 1963). Wood et al. (1999) demonstrated that in the LMC these multimode variables are definitely on the asymptotic giant branch (AGB). One group among the multi-period AGB variables has period ratios ranging from about 5 to 13 with the short period typical for a SR or Mira variable. The Wood et al. (1999) LMC period-luminosity diagram shows that they largely fall on a sequence in the period-luminosity diagram that is parallel to the sequence believed to be that of the fundamental-mode pulsators but at longer periods ranging from 250 to over 1000 days. Wood et al. (1999) have labeled this periodluminosity sequence ' $\mathrm{D}$ '. Since classical pulsation theory does not allow a period of radial pulsation longer than the fundamental mode, the nature of the long periods is unknown.

We have investigated field $M$ giants in our galaxy that have multiple modes with ratios of 5 to 13 , and thus are presumably sequence ' $D$ ' members. While some of these stars have been the targets of recent detailed photometric work by other observers, our investigation is spectroscopic in the near infrared range. In particular we were interested in determining if the long periods indicate that the giants are binaries.

Each program star has light variations with both a short and a long period. The short periods are in agreement with overtone stellar pulsation, and we have not investigated them further. A period search of the radial velocities was carried out for each program star, and a long period similar to that of the light variations 
was found for six stars. For each of these the long period from the velocities was adopted as the orbital period, and orbital elements were computed (Table 1). By fitting an orbit to the velocities we do not necessarily imply that these stars are spectroscopic binaries. Rather, this is an analysis technique to examine the nature of the velocity variations. Five of these six SR variables listed in

Table 1. Orbital elements.

\begin{tabular}{lrrrcrrr}
\hline Name & $\begin{array}{r}\mathrm{P} \\
\text { (days) }\end{array}$ & $\begin{array}{r}\gamma \\
\left(\mathrm{km} \mathrm{s}^{-1}\right)\end{array}$ & $\begin{array}{r}\mathrm{T} \\
(\mathrm{HJD})\end{array}$ & $\begin{array}{r}\mathrm{K} \\
\left(\mathrm{km} \mathrm{s}^{-1}\right)\end{array}$ & $\begin{array}{r}\omega \\
(\mathrm{deg})\end{array}$ & $\begin{array}{r}\mathrm{f}(\mathrm{M}) \\
\left(\mathrm{M}_{\odot}\right)\end{array}$ \\
\hline RS CrB & 328.3 & -80.9 & $2,451,770.9$ & 2.4 & 0.35 & 251 & 0.0004 \\
AF Cyg & 926.3 & -14.8 & $2,451,224.6$ & 1.8 & 0.08 & 58 & 0.0006 \\
X Her & 658.3 & -90.3 & $2,451,770.9$ & 1.6 & 0.32 & 319 & 0.0002 \\
g Her & 843.7 & 1.2 & $2,451,918.2$ & 2.3 & 0.37 & 246 & 0.0009 \\
V574 Oph & 690.0 & -35.7 & $2,451,540.0$ & 1.7 & 0.33 & 251 & 0.0003 \\
BI Peg & 548.4 & -28.2 & $2,451,368.9$ & 3.1 & 0.36 & 240 & 0.0013 \\
\hline
\end{tabular}

Table 1 have nearly identical values of $K$, e, and to a lesser extent of $\omega$ in the orbital fit. This strongly suggests that the velocity changes of these variables do not result from orbital motion. The existence of close binary companions to these stars also seems unlikely on the basis of the mass functions and the properties of the light curves. The similar shapes of the velocity curves imply that some, as yet unknown, type of pulsation is responsible for the velocity variations. Wood (2000) identified theoretically a family of "strange" pulsation modes that occur because of the interaction of stellar oscillations and convective energy transport. In his analyses the modes were highly damped, but other treatments of convection might produce unstable modes with periods similar to those found here.

Acknowledgments. This research was funded through grants by NASA (NCC 5-551 and NCC 5-96) and NSF (HRD 97-06268) to the Tennessee State University and by the Austrian Science Fund Project (P14365-PHY). NOAO is operated by the Association of Universities for Research in Astronomy, under cooperative agreement with the National Science Foundation. The observations would not have been possible without the loan of the NICMASS equipment to NOAO by M. Skrutskie.

\section{References}

Houk, N. 1963, AJ, 68, 253

Wood, P.R. 2000, in ASP Conf. Series, Vol. 203, The Impact of Large Scale Surveys on Variable Stars Research, eds. L. Szabados \& D. Kurtz, (San Francisco ASP), 379

Wood, P.R., Alcock, C., et al. 1999, in PASP, Vol. 191, Asymptotic Giant Branch Stars, eds. T. Le Berte, A. Lébre, \& C. Waelkens, (San Francisco ASP), 151 